\title{
DECOMPOSITION OF COMPLETE MULTIGRAPHS INTO STARS AND CYCLES
}

\author{
Fairouz Beggas, Mohammed Haddad \\ AND \\ Hamamache KhedDouci \\ LIRIS UMR 5205, CNRS, University of Lyon, \\ Claude Bernard Lyon 1 University \\ 43 Bd du 11 Novembre 1918, F-69622, Villeurbanne, France. \\ e-mail: fairouz.beggas@liris.cnrs.fr \\ mohammed.haddad@liris.cnrs.fr \\ hamamache.kheddouci@liris.cnrs.fr
}

\begin{abstract}
Let $k$ be a positive integer, $S_{k}$ and $C_{k}$ denote, respectively, a star and a cycle of $k$ edges. $\lambda K_{n}$ is the usual notation for the complete multigraph on $n$ vertices and in which every edge is taken $\lambda$ times. In this paper, we investigate necessary and sufficient conditions for the existence of the decomposition of $\lambda K_{n}$ into edges disjoint of stars $S_{k}$ 's and cycles $C_{k}$ 's.
\end{abstract}

Keywords: graph decomposition, complete multigraph, stars, cycles.

2010 Mathematics Subject Classification: $05 \mathrm{C} 70$.

\section{REFERENCES}

[1] A.A. Abueida and M. Daven, Multidesigns for graph-pairs of order 4 and 5, Graphs Combin. 19 (2003) 433-447. doi:10.1007/s00373-003-0530-3

[2] A.A. Abueida and M. Daven, Multidecompositions of the complete graph, Ars Combin. 72 (2004) 17-22.

[3] A.A. Abueida and T. O'Neil, Multidecomposition of $\lambda K_{m}$ into small cycles and claws, Bull. Inst. Combin. Appl. 49 (2007) 32-40.

[4] A.A. Abueida and C. Lian, On the decompositions of complete graphs into cycles and stars on the same number of edges, Discuss. Math. Graph Theory 34 (2014) $113-125$. doi:10.7151/dmgt.1719 
[5] B. Alspach and H. Gavlas, Cycle decompositions of $K_{n}$ and $K_{n}-I$, J. Combin. Theory, Ser. B 81 (2001) 77-99.

doi:10.1006/jctb.2000.1996

[6] D. Bryant, D. Horsley, B. Maenhaut and B.R. Smith, Cycle decompositions of complete multigraphs, J. Combin. Des. 19 (2011) 42-69. doi:10.1002/jcd.20263

[7] V. Chitra and A. Muthusamy, Symmetric Hamilton cycle decompositions of complete multigraphs, Discuss. Math. Graph Theory 33 (2013) 695-707. doi:10.7151/dmgt.1687

[8] S. Cichacz, Decomposition of complete bipartite digraphs and even complete bipartite multigraphs into closed trails, Discuss. Math. Graph Theory 27 (2007) 241-249. doi:10.7151/dmgt.1358

[9] H.-C. Lee and J.-J. Lin, Decomposition of the complete bipartite graph with a 1factor removed into cycles and stars, Discrete Math. 313 (2013) 2354-2358. doi:10.1016/j.disc.2013.06.014

[10] Z. Liang and J. Guo, Decomposition of complete multigraphs into crown graphs, J. Appl. Math. Comput. 32 (2010) 507-517. doi:10.1007/s12190-009-0267-0

[11] H.M. Priyadharsini and A. Muthusamy, $\left(G_{m}, H_{m}\right)$-multifactorization of $\lambda K_{m}$, J. Combin. Math. Combin. Comput. 69 (2009) 145-150.

[12] M. Šajna, Cycle decompositions III: Complete graphs and fixed length cycles, J. Combin. Des. 10 (2002) 27-78. doi: $10.1002 /$ jcd. 1027

[13] T.-W. Shyu, Decompositions of complete graphs into paths and cycles, Ars Combin. 97 (2010) 257-270.

[14] T.-W. Shyu, Decomposition of complete graphs into paths of length three and triangles, Ars Combin. 107 (2012) 209-224.

[15] T.-W. Shyu, Decomposition of complete graphs into cycles and stars, Graphs Combin. 29 (2013) 301-313. doi:10.1007/s00373-011-1105-3

[16] T.-W. Shyu, Decomposition of complete bipartite graphs into paths and stars with same number of edges, Discrete Math. 313 (2013) 865-871. doi:10.1016/j.disc.2012.12.020

[17] D. Sotteau, Decomposition of $K_{m, n}\left(K_{m, n}^{(*)}\right)$ into cycles (circuits) of length $2 k$, J. Combin. Theory, Ser. B 30 (1981) 75-81. doi:10.1016/0095-8956(81)90093-9

[18] M. Tarsi, Decomposition of complete multigraphs into stars, Discrete Math. 26 (1979) 273-278. doi:10.1016/0012-365X(79)90034-7 
[19] M. Tarsi, Decomposition of a complete multigraph into simple paths: Nonbalanced handcuffed designs, J. Combin. Theory, Ser. A 34 (1983) 60-70. doi:10.1016/0097-3165(83)90040-7

[20] R.M. Wilson, Decomposition of complete graphs into subgraphs isomorphic to a given graph, in: Proceedings of the 5th British Combinatorial Conference, Util. Math., Winnipeg, Congr. Numer. 15 (1976) 647-659.

[21] S. Yamamoto, H. Ikeda, S. Shige-eda, K. Ushio and N. Hamada, On clawdecomposition of complete graphs and complete bigraphs, Hiroshima Math. J. 5 (1975) 33-42.

Received 9 september 2014

Revised 23 December 2014

Accepted 23 December 2014 